\title{
COMPLICAÇÕES PÓS-OPERATÓRIAS EM PACIENTES SUBMETIDOS À ABDOMINOPLASTIA ISOLADA E COMBINADA À OUTRAS CIRURGIAS DO ABDOME
}

\author{
POSTOPERATIVE COMPLICATIONS IN PATIENTS SUBMITTED TO ISOLATED \\ ABDOMINOPLASTY ALONE OR ASSOCIATED TO OTHER ABDOMINAL SURGERIES
}

\author{
Carlos Alberto Porchat, TCBC-RJ ${ }^{1}$; Elizabeth Gomes dos Santos, TCBC-RJ'2; \\ Guilherme Pinto Bravo Neto, TCBC-RJ ${ }^{3}$
}

\begin{abstract}
RESUMO: Objetivo: A realização de abdominoplastias associadas a outras cirurgias da parede ou da cavidade abdominal, embora atrativa, é motivo de controvérsias. O objetivo deste trabalho é avaliar o grau de morbidade e mortalidade destas associações. Método: Foram estudados retrospectivamente 75 pacientes com indicação de abdominoplastia dos quais 39 submeteram-se a abdominoplastia isoladamente (grupo 1) e 36 à associação de abdominoplastia a outras cirurgias do abdome (grupo 2), como correção de hérnias, histerectomias e colecistectomias. Foram analisados os diversos fatores capazes de interferir na evolução do paciente, como doenças pré-existentes, assim como as complicações pós-operatórias. Resultados: As complicações observadas foram: seroma (grupo 1: $2.6 \%$, grupo 2: $25 \%$,), epidermólise (grupo 1: $12.82 \%$, grupo 2: $5.55 \%$ ), deiscência de sutura (grupo 1: $5.12 \%$, grupo 2: $5.55 \%$ ), infecção da ferida operatória (grupo 1: $0 \%$, grupo 2: $8.33 \%$ ) e hematoma (grupo 1: 0\%, grupo 2: 5.55\%). Não houve diferença estatisticamente significante entre os dois grupos no que se refere a complicações pós-operatórias, exceto quanto ao seroma $(\mathrm{p}=0,009)$. Conclusão: Concluímos que não houve aumento significativo da morbidade e da mortalidade dos pacientes submetidos a cirurgias combinadas quando comparados aos pacientes submetidos à abdominoplastia isoladamente (Rev. Col. Bras. Cir. 2004; 31(6): 368-372).
\end{abstract}

Descritores: Complicações pós-operatórias; Abdome/Cirurgia; Cirurgia plástica.

\section{INTRODUÇÃO}

Os cirurgiões gerais e os ginecologistas são cada vez mais solicitados por seus pacientes para, em um mesmo ato operatório, associar uma cirurgia estética ao tratamento de uma doença da parede ou da cavidade abdominal. A idéia de diminuir os custos com uma só internação e de submeter os pacientes a uma só anestesia é extremamente atrativa. No entanto, muitos cirurgiões ainda se sentem temerosos em aceitar esta combinação cirúrgica por receio de aumentar a morbidade e a mortalidade do procedimento.

O objetivo deste trabalho é analisar através de estudo retrospectivo, o índice de complicações pós-operatórias de pacientes submetidos à abdominoplastia associada a outros procedimentos da parede ou da cavidade abdominal e compará-lo àquele de pacientes submetidos à abdominoplastia isoladamente.

\section{MÉTODO}

Foi realizada análise retrospectiva de 75 pacientes consecutivos, submetidos a abdominoplastia, isoladamente, em 39 pacientes (grupo 1), e à abdominoplastia combinada à outra cirurgia no abdome em 36 pacientes (grupo 2). As cirurgias foram realizadas no Hospital Universitário Clementino Fraga Filho - UFRJ e em clínica particular.
Todos os pacientes operados foram classificados como ASA I e II segundo a tabela da American Society of Anesthesiologists (Phisical Status Scale). A anestesia utilizada foi a anestesia geral ou bloqueio espinhal associado à sedação venosa.

A abdominoplastia foi indicada para pacientes que apresentavam excesso de pele, nos quais havia possibilidade de ressecção de todo o retalho infra-umbilical e em alguns casos ressecção vertical combinada.

A revisão dos prontuários teve como base um protocolo que foi aplicado a cada paciente, onde as seguintes variáveis foram analisadas: faixa etária, sexo, tipos de associação cirúrgica, tipos de complicações, relação entre cirurgias e complicações, relação entre complicações e co-morbidades, relação entre tempo de internação e complicações, relação entre tipos de cirurgias e tempo de internação, relação entre o uso da tela de Marlex e complicações.

Após a distribuição dos dados, estes foram submetidos à análise estatística pelo programa "Statistical Analysis System". Foi utilizado o Teste de Qui-Quadrado ou o Teste Exato de Fisher para a comparação de proporções entre dois grupos. Para a comparação da idade média entre dois grupos foi utilizado o Teste t de Student. Na comparação da duração da cirurgia e do tempo de internação foi utilizado o Teste de Mann-Whitney. O nível de significância adotado foi de $5 \%$.

1. Membro Titular da Sociedade Brasileira de Cirurgia Plástica; Cirurgião Plástico do Hospital Clementino Fraga Filho - Universidade Federal do Rio de Janeiro.

2. Cirurgiã do Serviço de Cirurgia Geral do Hospital Clementino Fraga Filho - Universidade Federal do Rio de Janeiro.

3. Professor Adjunto do Departamento de Cirurgia Geral da Universidade Federal do Rio de Janeiro.

Recebido em 10/05/2004

Aceito para publicação em 14/06/2004

Trabalho realizado no Hospital Universitário Clementino Fraga Filho - Universidade Federal do Rio de Janeiro; Tese de Mestrado do Curso de PósGraduação do Departamento de Cirurgia da Faculdade de Medicina da Universidade Federal do Rio de Janeiro. 


\section{RESULTADOS}

Setenta e um pacientes eram de sexo feminino $(94,7 \%)$ e quatro do sexo masculino $(5,3 \%)$. O grupo 1 era formado por 36 mulheres e três homens e o grupo 2 por 35 mulheres e um homem.

A idade dos pacientes variou de 28 a 84 anos, com média de 46,75 anos. A média de idade do grupo 1 foi de 45,5 anos e do grupo 2 de 48 . Não houve diferença significativa em relação à idade entre os dois grupos $(\mathrm{p}=0,54)$.

As doenças associadas observadas foram: hipertensão $(24,5 \%)$, diabetes $(9,3 \%)$, cardiopatia isquêmica (4\%), asma (4\%) e hipotiroidismo (1,3\%). A maioria dos pacientes $(58,8 \%)$ não apresentava doenças associadas. Alguns pacientes eram portadores de mais de uma doença associada.

As indicações cirúrgicas, além da lipodistrofia abdominal, podem ser vistas na Tabela 1. Predominaram os defeitos da parede abdominal como hérnias e eventrações. As cirurgias associadas à abdominoplastia encontram-se na Tabela 2 .

Entre as complicações observadas sobressai-se o seroma , que foi estatisticamente mais frequente nas cirurgias combinadas (Tabela 3). Em 11 pacientes foi necessária a utilização de tela de Marlex para correção de hérnias e eventrações. Na comparação entre a utilização da tela de Marlex e da presença de seroma, hematoma, infecção de ferida operatória, epidermólise e deiscência, a única complicação estatisticamente significativa foi a presença de seroma $(p=0,009)$. Não foi encontrada relação entre seroma e a presença de outras doenças clínicas pré-existentes.

Tabela 1 - Indicações de cirurgias associadas.

\begin{tabular}{lr}
\hline Doenças cirúrgicas associadas & n \\
\hline Hérnia Incisional / Eventração & 19 \\
Hérnia de Spiegel & 1 \\
Hérnia Inguinal & 3 \\
Hérnia Umbilical & 2 \\
Ferida em granulação & 3 \\
Mioma & 13 \\
Colelitíase & 2 \\
\hline
\end{tabular}

Tabela 2 - Procedimentos cirúrgicos associados à abdominoplastia.

\begin{tabular}{lr}
\hline Procedimentos & n \\
\hline Histerectomia & 11 \\
Hernioplastia umbilical & 2 \\
Hernioplastia inguinal & 2 \\
Hernioplastia de Spiegel & 1 \\
Hernioplastia incisional & 17 \\
Hernioplastia inguinal + colecistectomia & 1 \\
Hernioplastia Incisional + histerectomia & 1 \\
Hernioplastia Incisional + histerectomia & \\
+ colecistectomia & 1 \\
\hline
\end{tabular}

A duração da cirurgia variou de 60 a 360 minutos. A duração média na abdominoplastia isolada foi de 180 minutos e quando houve cirurgia associada, a duração média foi de 220 minutos. Houve diferença significativa entre os dois gru$\operatorname{pos}(\mathrm{p}=0,0001)$.

Foi encontrada diferença significativa entre os dois grupos em relação ao tempo de internação $(p=0,009)$. O tempo de máximo de internação foi de 74 dias em cirurgia combinada e de 43 dias em abdominoplastia isolada. A internação mínima foi de dois dias em ambos os grupos e a média foi de quatro dias no grupo 1 e de cinco dias no grupo 2. A única relação entre o tempo de internação e doenças clínicas préexistentes em que houve significância estatística $(\mathrm{p}=0,009)$ foi quanto à presença de hipertensão arterial.

\section{DISCUSSÃO}

A lipectomia abdominal foi inicialmente descrita por Demars e Marx em $1890^{1-4}$. Este termo foi posteriormente modificado para abdominoplastia e refere-se às cirurgias em que existe ressecção de pele e tecido subcutâneo em excesso. Pode estar associada ou não a vários outros procedimentos e técnicas em que se atua sobre a musculatura (como rotação e plicatura dos músculos oblíquos externos), aponeurose, e com procedimentos complementares no subcutâneo (como lipoaspiração ou ressecções segmentares) ${ }^{3}$. Segundo Grazer ${ }^{5}$, Gaudet e Morestin, em 1905, relataram ressecção de pele e gordura com preservação da cicatriz umbilical no tratamento de grandes hérnias da parede abdominal e em 1911, Morestin 6 publicou três casos de dermolipectomia por excisões elípticas transversas. Em 1967, Pitanguy ${ }^{7}$ publicou análise de 300 casos consecutivos de abdominoplastia, comentando a técnica, resultados e complicações. O mesmo autor $^{8}$, em 1975, analisou a abdominoplastia em 539 casos. Ousterhout $^{9}$ em 1990, preconizou a associação de abdominoplastia à lipoaspiração da região abdominal e flancos. Pontes ${ }^{10}$ em 1965 e Franco ${ }^{11}$ em 1971, publicaram os primeiros artigos no Brasil sobre a associação da abdominoplastia a diversas cirurgias do abdome, indicando a viabilidade do procedimento em conjunto. Grazer et $a l .^{2}$ em 1977, descreveu pesquisa multicêntrica avaliando 10490 abdominoplastias isoladas, reportando com destaque as complicações do procedimento, dentre as quais se sobressaem: infecção de ferida, deiscência de sutura e

Tabela 3 - Complicações pós-operatórias.

\begin{tabular}{lrcc}
\hline Complicações & n & Grupo 1 & Grupo 2 \\
\hline Seroma & 10 & $2,56 \%$ & $25 \%$ \\
Hematoma & 2 & $0 \%$ & $5,55 \%$ \\
Infecção de ferida operatória & 3 & $0 \%$ & $8,33 \%$ \\
Epidermólise & 7 & $12,82 \%$ & $5,55 \%$ \\
Deiscência de sutura & 4 & $5,12 \%$ & $5,55 \%$ \\
\hline
\end{tabular}

Houve diferença estatística entre os dois grupos apenas na presença de seroma $(p=0,009)$; As porcentagens são referentes ao total de complicações. 
tromboflebite profunda. Na última década, muitos cirurgiões ${ }^{3,12,13}$ manifestaram suas preocupações com as associações e descrevem suas complicações como infecção, embolia pulmonar, perda sanguínea e endometrioma da parede abdominal. Outros autores ${ }^{14-17}$ indicaram a abdominoplastia associada a intervenções ginecológicas, ressaltando que este método facilitaria o procedimento intracavitário, através da ampliação do campo cirúrgico. A associação com o tratamento de hérnias ou eventrações também passou a ser descrita e indicada por vários autores $^{18-21}$. Gemperli ${ }^{1}$, em 1992, verificou em 103 pacientes com associação de abdominoplastias a outras cirurgias da cavidade abdominal que o índice de complicações é muito baixo, quando há indicação criteriosa e execução cuidadosa.

A abdominoplastia é uma das cirurgias plásticas mais realizadas em todo o mundo. É a correção do excesso do tecido cutâneo e adiposo através de sua ressecção. Pode ser associada ou não a procedimentos sobre a musculatura, como rotação ou plicatura dos músculos oblíquos externos, aponeuroses abdominais ou a lipoaspirações. ${ }^{8,9,22-24}$. Apesar do número de abdominoplastias em homens estar aumentando, o maior número de pacientes ainda é do sexo feminino. Talvez devido ao preconceito masculino em relação à cirurgia plástica e ao fato de ocorrer uma flacidez abdominal mais acentuada em algumas mulheres após a gravidez. Estes dados embora não relatados especificamente em outros trabalhos envolvendo grandes séries de pacientes $^{2,7}$ são sugeridos pela referência constante ao sexo feminino.

Em relação à idade, encontramos uma grande variação (28 a 84 anos), sendo que em relação aos dois grupos não houve diferença estatística significante. A média de idade foi de 48 anos. Nesta faixa etária, encontramos maior frequiência de pacientes com lipodistrofia do abdome e um número maior de outras doenças abdominais associadas. Fran$\mathrm{co}^{11}$, Mohamad $^{25}$, Floros ${ }^{26}$ e Pitanguy ${ }^{7,8}$ em suas séries encontraram dados semelhantes. Alguns pacientes eram portadores de doenças clínicas associadas. Isso deve ser levado em consideração como causa de aumento da morbidade no per e no pós-operatório. Dentre elas a mais comum foi a hipertensão arterial, presente em 18 pacientes $(24,5 \%)$. A hipertensão arterial foi a única doença pré-existente que influenciou significativamente no período de internação. Não houve evidências de que doenças clínicas interferissem na evolução pós-operatória em ambos os grupos. Todos os pacientes operados foram classificados como ASA I ou II, o que significa que eram pacientes com baixo risco de complicações pós-operatórias.

Em relação à doença abdominal associada à indicação de abdominoplastia, a mais freqüente foi a hérnia incisional, dado este coincidente com as publicações no Brasil, de Carreirão et $a l^{19}$ e Pontes ${ }^{10}$ que, descrevendo sobre esta associação, sugerem que a cura das hérnias incisionais é facilitada pela plástica abdominal, a qual promove um campo cirúrgico mais adequado. Segundo os autores, este procedimento facilita a exposição e o tratamento do defeito herniário. Sensoz et al ${ }^{21}$, em 1997, idealizou técnica para correção de alguns tipos de hérnias da parede abdominal com a utilização de um retalho inferior do abdome (tecido que é usualmente ressecado) para o reforço da parede abdominal. Esta técnica não foi utilizada em nossos casos, mas achamos que a mesma poderia ser uma opção alternativa quando há dificuldade na aquisição de telas de material aloplástico. Em nossa série, a histerectomia foi o segundo procedimento associado mais comum e foi indicada pela presença de miomas em todos os casos, como observado também por Franco ${ }^{11}$, Hester et $\mathrm{l}^{3}$, Gemperli et al ${ }^{1}$ e Shull et al ${ }^{27}$. Cosin et al ${ }^{28}$ sugerem que a associação de cirurgias pélvicas com abdominoplastias facilita o acesso e previne complicações da ferida operatória. Segundo os autores, o abdome pendular, cria uma área de dobra da pele com aumento de umidade, que favorece a proliferação bacteriana, levando a uma incidência maior de infecção da ferida. Como complicação da associação de histerectomia com abdominoplastia, Matthes et al ${ }^{12}$ relataram o aparecimento de endometrioma da parede abdominal cinco anos após a cirurgia. Em alguns pacientes houve a combinação de várias cirurgias como colecistectomia, histerectomia e hernioplastia com a abdominoplastia.

Houve concordância de todos os cirurgiões que participaram das cirurgias combinadas deste estudo, que o campo cirúrgico exposto de uma maneira mais ampla pela abdominoplastia, facilitou a correção da doença intra-abdominal. O maior motivo de controvérsias quanto à indicação da combinação de cirurgias é o temor do aumento da morbidade e mortalidade. Vários tipos de complicações são relatados em abdominoplastias isoladas: hematoma, seroma, infecção de ferida operatória, deiscência, necrose do retalho abdominal, celulite da parede abdominal, epidermólise, embolia pulmonar, tromboflebite, choque e outras ${ }^{29}$. Nas cirurgias associadas, estas complicações tenderiam a ser em número maior devido ao tempo cirúrgico aumentado, maior perda de sangue, manipulação maior dos tecidos e tempo de internação mais prolongado.

Os seromas (coleções de plasma entre a aponeurose e camada gordurosa) representaram $38 \%$ e os hematomas $8 \%$ do total de complicações. Estes diagnósticos foram feitos clinicamente, através da visualização de abaulamentos e pela palpação, seguidos de punções. A presença de seromas e de hematomas apesar de relativamente alta foi representada por coleções de tamanho pequeno e que foram absorvidos com o tempo sem alterar a evolução normal do pós-operatório. Mohamed $e t a l^{25}$, usaram o ultra-som no pós-operatório de pacientes submetidos a abdominoplastia, algumas associadas ao tratamento de hérnias, e verificaram uma incidência de $51,8 \%$ de coleções fluidas subcutâneas (hematomas e seromas). Na nossa série, o seroma ocorreu em $2.56 \%$ dos pacientes do grupo 1 e em $25 \%$ dos pacientes do grupo 2, sendo a única que teve significância estatística $(\mathrm{p}=0,009)$. $\mathrm{Na}$ análise do grupo 2 houve maior incidência de seroma nas cirurgias associadas com o tratamento de hérnias incisionais $(16,66 \%$ do grupo de cirurgias associadas). Verificamos que isso ocorreu em associação ao uso freqüente de tela de Marlex neste tipo de cirurgia. A análise estatística dos dados quando se avaliou a utilização da tela com outras complicações revelou que o 
seroma foi a única que teve representação estatística significativa $(p=0,009)$.

O hematoma representou $8 \%$ no total das complicações. Não foi encontrado em abdominoplastias isoladas, e nas associadas estava presente quando houve o tratamento de hérnias. Não houve necessidade de intervenção cirúrgica para o tratamento dos seromas e hematomas. Os mesmos foram tratados através de punções em regime ambulatorial sem interferir no resultado final das cirurgias.

A segunda complicação mais freqüente foi o sofrimento superficial da pele na linha de sutura, a epidermólise (27\%) e foi estatisticamente semelhante nos dois grupos. Este tipo de complicação geralmente está associado à tensão ou traumatismo do retalho. O tratamento foi feito com curativos e pomadas com desbridantes químicos e emolientes.

A deiscência da ferida operatória pode ocorrer por infecção ou pelo excesso na ressecção, com tensão no retalho, isquemia e interferência na cicatrização. Em nossa casuística encontramos $5,12 \%$ de deiscência da ferida em abdominoplastia isolada e 5,55\% nas cirurgias combinadas, diferença sem significância estatística. Estes dados são semelhantes ao estudo de Grazer e Goldwyn ${ }^{2}$, que ainda chamam a atenção para que este índice é maior entre os cirurgiões com pouco tempo de experiência na especialidade.

A infecção da ferida operatória é uma das complicações mais temidas porque pode colocar em risco não só o resultado das cirurgias como vida dos pacientes. Vários fatores interferem no aparecimento da infecção ${ }^{30,31}$. Três são considerados de grande importância na avaliação do risco de infecção do sítio cirúrgico: o potencial de contaminação da ferida operatória, que em cirurgias limpas e potencialmente contaminadas é pequeno e nas cirurgias contaminadas e infectadas é muito grande; o risco cirúrgico segundo critérios da Associação Americana de Anestesistas (ASA), em que pacientes classificados como ASA I ou II são de baixo risco para aparecimento de complicaçoes pós-operatorias e pacientes ASA III ou IV de alto risco; e a duração da cirurgia, segundo critérios estatísticos para cada tipo de procedimento. Desta forma observamos que, nossos pacientes puderam ser classificados como de baixo risco quanto ao potencial de contaminação da ferida operatória (todas a cirurgias foram limpas ou potencialmente contaminadas), e quanto ao risco cirúrgico (todos os pacientes foram classificados como ASA I ou II). A única variável capaz de aumentar o risco de infecção cirúrgica foi a duração da cirurgia que foi de 180 minutos no grupo 1 e de 220 minutos no grupo 2, uma diferença estatisticamente significante, porém representadas por um prolongamento da duração de cirurgia de apenas 40 minutos em média. O índice de infecção de 8,33\% em cirurgias combinadas, no entanto, não teve significância estatística quando comparado a $0 \%$ em abdominoplastias isoladas.
A obesidade é citada como um fator de aumento no índice de infecção por Savage $\mathrm{e}^{4} \operatorname{Voss}^{13}$. Em nossos casos, o paciente que desenvolveu infecção mais grave da ferida e celulite do retalho era obeso.

O déficit na vascularização dos retalhos e da drenagem linfática como sendo causa de desenvolvimento celulite, foi relatado por alguns autores ${ }^{32}$ principalmente nos casos em que se utiliza a técnica de incisões combinadas (em T invertido). Em nossos casos não constatamos qualquer relação com este tipo de técnica e a presença de infecção ou deiscências.

A presença de infecção na ferida aumentou muito o tempo de internação em pacientes do grupo 2. Acreditamos no entanto, que este fato esteja relacionado não só com a complicação infecciosa, mas também, com dificuldades sócioeconômicas de alguns pacientes operados no Hospital Universitário.

Em séries de pacientes analisadas por outros autores, foram encontrados outros tipos de complicações, como embolia pulmonar e choque hipovolêmico ${ }^{3}$, não observadas em nossa casuística. Acreditamos que a técnica cirúrgica meticulosa, a deambulação precoce (nas primeiras 24 horas de pós-operatório) e seleção criteriosa de pacientes foram fatores importantes na prevenção destas complicações.

O objetivo de qualquer tratamento cirúrgico deve ser sempre um resultado eficiente e sem complicações. É fundamental um amplo entendimento entre os cirurgiões das diferentes especialidades envolvidos em uma mesma cirurgia. Os mesmos devem estar a par das técnicas e táticas usadas e estar de acordo entre eles, o que diminui a incidência de complicações. Nenhum médico deve sentir-se compelido a realizar procedimentos, premidos por seus pacientes, sem que haja uma cuidadosa análise da indicação cirúrgica e do estado clínico dos pacientes. Pode ser tentador tanto para o paciente, quanto para o cirurgião a realização de cirurgias combinadas. A economia em taxas extras de centro cirúrgico, despesas médicas e recobrar-se de duas operações simultaneamente sem a necessidade de se afastar do trabalho ou usar férias duas vezes, são ponderações a favor das cirurgias combinadas. $\mathrm{O}$ envolvimento dos pacientes na decisão da cirurgia, tornando-os conscientes de todas as etapas e possíveis complicações, deve ser parte da cirurgia para um melhor preparo pré-operatório e para um melhor resultado final.

Concluímos que a associação de abdominoplastia com outras cirurgias do abdome é uma alternativa viável tecnicamente, em que o pequeno aumento da morbidade não contra indica estes procedimentos associados. $\mathrm{O}$ aumento estatisticamente significante de seroma nas cirurgias combinadas não interferiu no resultado final das cirurgias e esteve relacionado à utilização da tela de Marlex. Houve aumento estatisticamente significante da duração das cirurgias combinadas que, no entanto, não resultou em aumento das taxas de infecção. 


\begin{abstract}
Background: The association of abdominoplasty with other surgeries of the abdominal wall and cavity is an attractive approach although it has been a matter of controversies. Our objective is to evaluate the morbidity and mortality of these procedures. Methods: We studied retrospectively 75 patients with indication for abdominoplasty. Thirty nine of them were submitted to abdominoplasty alone (group 1) and 36 were associated with other abdominal surgeries (group2), as hernioplasties, hysterectomies or cholecystectomies. Results: The complications were seroma (group 1: 2.56\%, group 2: 25\%), epidermolisis (group 1: 12.82\%, group 2: 5.55\%), suture dehiscence (group 1: 5.12\%, group 2: $5.55 \%$ ), wound infection (group 1: 0\%, group 2: $8.33 \%$ ) and haematoma (group 1: 0\%, group 2: 5.55\%). There was statistical difference only in the presence of seroma ( $p=0.009)$. Conclusions: There was no significant increase in morbidity and mortality when abdominoplasty was combined to other abdominal surgeries compared to isolated abdominoplasty.
\end{abstract}

Key words: Postoperative Complications; Abdomen/Surgery; Plastic surgery.

\section{REFERÊNCIAS}

1. Gemperli R, Neves RI, Tuma P, et al. - Abdominoplasty combined with other intraabdominal procedures. Ann Plast Surg, 1992, 29(1):18-22.

2. Grazer FM, Goldwyn RM - Abdominoplasty assessed by survey, with enphasis on complications. Plast Reconst Surg, 1977, 59(4):513-517.

3. Heste TR, Baird W, Bostwick J, et al. - Abdominoplasty combined with other major surgical procedures: safe or sorry? Plast Reconst Surg, 1989, 83(6):997-1004

4. Savage RC - Abdominoplasty combined with other surgical procedures. Plast Reconst Surg, 1982, 70(4):437-443, 1982

5. Grazer FM - "Abdominoplasty". In McCarthy JG - Plastic Surgery. Saunders, Vol. 6, pp.3929-3963, 1990.

6. Morestin A - La restauration de la paroi abdominale par réssection etendue des teguments et la graisse sous-cutanée et le plissements des aponévroses superficielles envisagécomme complément de la cure radicale des hernies umbilicales. Thése, Paris, 1911, apud Grazer, F.M. Abdominoplasty, McCarthy, J.G. Plastic Surgery, Saunders, Vol. 6, 3929 - 3963, 1990.

7. Pitanguy I - Abdominal lipectomy: an aproach to it through an analysis of 300 consecutive cases. Plast Reconst Surg, 1967, 40(4):384-391.

8. Pitanguy I - Abdominal lipectomy. Clin Plast Surg, 1975, 2(3):401-410.

9. Ousterhout D - Combined suction-assited lipectomy, surgical lipectomy, and surgical abdominoplasty. Ann Plast Surg, 1990, 24(2):126-133.

10. Pontes R - Plástica abdominal: importância de sua associação à correção das hérnias incisionais. Rev Bras Cir, 1965, 52(1):85-88.

11. Franco T - A associação de abdominoplastia a cirurgias intracavitárias. Ver Bras Cir, 1971, 61(1):5-17

12. Matthes G, Zabel DD, Nastala CL, et al. - Endometrioma of the abdominal wall following combined abdominoplasty and histerectomy: case report and review of the literature. Ann Plast Surg, 1998, 40(6):672-675

13. Voss SC, Sharp HC, Scott JR - Abdominoplasty combined with gynecologic surgical procedures. Obstet Gynecol, 1986, 67(2):181-185

14. Castro CC, Salema R, Aboudib Jr JH, et al. - Análise da associação entre abdominoplastia e cirurgias ginecológicas. J Bras Ginecol, 1991,101(10):447-449.

15. Castro CC - Estudo da associação cirúrgica da plástica de abdome com procedimentos ginecológicos. J Bras Ginecol, 1977, 83(3):137-142.

16. Perry AW - Abdominoplasty combined with total abdominal histerectomy. Ann Plast Surg, 1986,16(2):121-124.
17. Pretorius RG, Matory WE, LaFontaine D - Management of massive ovarian tumors. Surg Gynecol Obstet, 1989, 169(6):532-536.

18. Bang RL, Behbehani IA - Repair of large, multiple, and recurrent ventral hernias: an analysis of 124 cases. Eur J Surg, 1997, 163(2):107-114.

19. Carreirão S, Correa WE, Dias LC, et al. -Treatment of abdominal wall eventrations associated with abdominoplasty techniques. Aesth Plast Surg, 1984, 8(3):173-179.

20. Hughes KC, Weider L, Fischer J, et al. - Ventral hernia repair with simultaneous panniculectomy. Am Surg, 1996, 62(8):678-681.

21. Sensõz O, Arifoglu K, Koçer U, et al. - A new approach for the treatment of recurrent large abdominal hernias: the overlap flap. Plast Reconst Surg, 1997, 99(7):2074-2078.

22. Ashbell TS - Abdominoplasty and combined procedures. Plast Reconst Surg, 1987, 79(2):316.

23. Bozola AR, Psillakis JM - Abdominoplasty: a new concept and classification for treatment. Plast Reconst Surg, 1988, 82(6):983993.

24. Nahas FX, Augusto SM, Ghelfond C - Should diastasis recti be corrected? Aesthetic Plast Surg, 1997, 21(4):285-289.

25. Mohammad JA, Warnke PH, Stravaky W - Ultrasound in the diagnosis and management of fluid collection complications following abdominoplasty. Ann Plast Surg, 1998, 41(5):498-502.

26. Floros C, Davis PK - Complications and long-term results following abdominoplasty: a retrospective study. Br J Plast Surg, 1991, 44(3):190-194.

27. Shull BL, Verheyden CN - Combined plastic and gynecological surgical procedures. Ann Plast Surg, 1988, 20(6):552-557.

28. Cosin JA, Powell JL, Donovan JT, et al. - The safety and efficacy of extensive abdominal panniculectomy at the time of pelvic surgery. Gynecol Oncol, 1994, 55(1):36-40

29. Hunter GR, Crapo RO, Broadbent TR, et al. - Pulmonary complication following abdominal lipectomy. Plast Reconst Surg, 1983, 71(6):809-817

30. Bravo Neto GP - Infecção cirúrgica. Dissertação (Mestrado em Cirurgia Geral). Rio de Janeiro. Universidade Federal do Rio de Janeiro, 1988, 166 p. 31 - Bravo Neto GP - Infecção em cirurgia: clínica cirúrgica fundamentos teóricos e práticos. Rio de Janeiro - Atheneu, 2000

32. Kronowitz SJ, Weisstein J, Greenstein R - Does abdominoplasty predispose to erysipelas of the abdominal wall? Ann Plast Surg, 1998, 41(4):453-454

Endereço para correspondência:

Carlos Porchat

R. Voluntários da Pátria 190 s. 1016/1017

22270-010- Rio de Janeiro - RJ

Email: porchat@wnetrj.com.br 